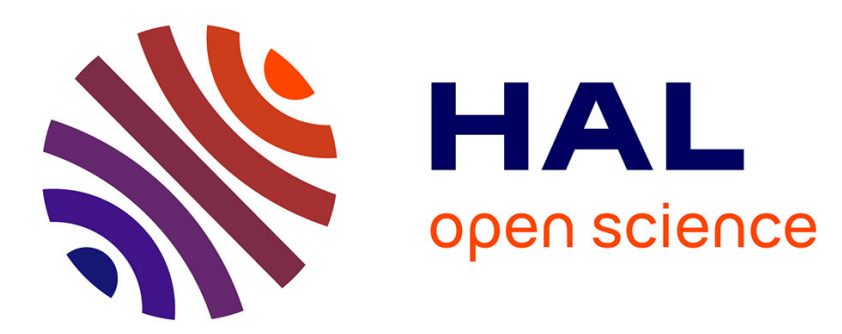

\title{
Un dispositif d'écriture collaborative au service de la réflexivité en formation d'enseignants
}

\author{
Marie-Laure Elalouf, Stéphanie Martel, Pierre Moinard
}

\section{To cite this version:}

Marie-Laure Elalouf, Stéphanie Martel, Pierre Moinard. Un dispositif d'écriture collaborative au service de la réflexivité en formation d'enseignants. Études de linguistique appliquée: revue de didactologie des langues-cultures, 2016, 185, pp.477-490. hal-02980147

\section{HAL Id: hal-02980147 https://hal-cyu.archives-ouvertes.fr/hal-02980147}

Submitted on 11 Feb 2022

HAL is a multi-disciplinary open access archive for the deposit and dissemination of scientific research documents, whether they are published or not. The documents may come from teaching and research institutions in France or abroad, or from public or private research centers.
L'archive ouverte pluridisciplinaire HAL, est destinée au dépôt et à la diffusion de documents scientifiques de niveau recherche, publiés ou non, émanant des établissements d'enseignement et de recherche français ou étrangers, des laboratoires publics ou privés. 


\section{Un dispositif d'écriture collaborative au service de la réflexivité en formation d'enseignants Marie-Laure ELALOUF, Stéphanie MARTEL et Pierre MOINARD}

L'article met en perspective une expérience de formation en ligne, menée en 2009-10 (ELA n ${ }^{\circ} 60$ ) et un dispositif proposé en 2015-16 à l'ÉSPE de l'académie de Versailles à des stagiaires de Lettres. Il leur est demandé d'élaborer un travail scientifique de nature réflexive. L'enjeu de ce travail a conduit des formateurs à concevoir un accompagnement serré du processus d'écriture, alternant des séances en présentiel et des moments d'échanges à distance sur ETHERPAD, logiciel associant une messagerie instantanée à un document co-élaboré. L'analyse des différents pads montre que la dynamique des interactions permet à ces débutants d'évoluer sur une échelle de réflexivité.

À partir d'une expérience d'écriture collaborative à distance menée avec des professeurs stagiaires de lettres au cours de l'année 2016, cet article interroge le rôle des interactions écrites asynchrones dans les manifestations de retours sur la pratique enseignante. Les analyses qualitatives de différentes expressions de la réflexivité à travers une séquence d'échanges qui en constituent la $3^{\mathrm{e}}$ partie sont préparées par deux éclairages complémentaires : d'abord une comparaison avec une expérimentation plus ancienne de formation à distance ; puis une définition de l'écrit professionnel et des modalités de son accompagnement.

Le dispositif étudié dans cet article fait écho à une expérience de formation à distance de professeurs de Lettres menée en 2009 à l'université de Cergy-Pontoise. Pendant un trimestre, un groupe de 19 néotitulaires ${ }^{1}$ volontaires et leur formateur avaient travaillé sur un environnement numérique de travail (ENT) conçu spécifiquement pour favoriser les échanges collaboratifs (ÉLA n ${ }^{\circ} 160, \mathrm{Ahr}$ et al.). Les formations de 2009 et 2016 présentent des analogies : elles s'adressent à un public semblable, répondent à des visées analogues et exploitent les possibilités offertes par des situations de communication écrite et multiadressée ${ }^{2}$. Pourtant les tâches, les modalités d'accompagnement du travail diffèrent ${ }^{3}$ ainsi que les outils de communication et leurs usages.

Précisons la comparaison.

\section{MISE EN PERSPECTIVE DE DEUX FORMATIONS À DISTANCE}

\section{1. De la plateforme au pad ${ }^{4}$}

La principale différence des deux séquences de formation à distance concerne la nature de l'espace numérique. L'expérimentation de 2009 s'appuyait sur une plateforme regroupant différentes fonctionnalités dans une même interface numérique qui cherchait à créer une impression de cohabitation chez les usagers. Le premier travail avait consisté à définir et paramétrer à partir de l'ENT de l'IUFM, grâce à l'aide d'un informaticien, une agrégation spécifique de fonctionnalités accessibles à partir d'une page d'accueil. Cet espace de collaboration associait des bibliothèques (des espaces de dépôt et de réception de document), des forums et des salons (c'est-à-dire des espaces de travail collaboratif en équipe de trois étudiants). En l'occurrence, la succession des tâches demandées aux différents moments du scénario de formation se lisait dans l'architecture même d'un espace virtuel complexe : prendre connaissance des questions qui orientent la réflexion du groupe sur la page d'accueil ; s'approprier des documents ressources produits par le tuteur ou les autres stagiaires dans des bibliothèques; participer aux échanges asynchrones sur le forum; offrir ses productions à partir des bibliothèques de dépôt.

En 2016, le choix du pad se distingue nettement de l'option intégrative (voire totalisante) de la plateforme : cette modalité de travail est proposée à des enseignants qui ne sont pas encore titulaires et

\footnotetext{
${ }^{1}$ Ces professeurs titularisés recevaient une formation obligatoire lors de leur première année d'exercice en contrepartie d'une décharge de service de deux heures hebdomadaires.

${ }^{2}$ Dans une situation de communication multiadressée, chaque message prend implicitement et nécessairement comme destinataires l'ensemble des participants aux échanges.

${ }^{3}$ La formation proposée en 2009 s'effectuait quasi entièrement à distance.

${ }^{4}$ On appelle pad (Packet Assembler and Desassembler) un éditeur de textes collaboratifs en ligne et les textes ainsi édités.
} 
dont l'expérience d'enseignement est souvent limitée : fonctionnaires-stagiaires, ils sont en responsabilité à mi-temps dans des classes de collège ou de lycée et en formation en présentiel deux jours par semaine à l'ÉSPÉ. ETHERPAD, utilisé dans le cadre de l'UE recherche, est avant tout un outil d'écriture en collaboration, associant pendant le temps de la co-production d'un texte deux fonctionnalités complémentaires : un traitement de texte actif simultanément pour tous les participants inscrits et un chat. Pourtant, dès qu'il est utilisé, le pad produit un espace commun. Les analyses de la $3^{\mathrm{e}}$ partie de l'article montreront comment le texte co-élaboré en vient à constituer le lieu même de la rencontre des participants.

De la plateforme au pad, le rapport de l'agir scriptural et de l'espace numérique s'est inversé. La plateforme constitue un environnement construit pour favoriser la collaboration par l'écriture alors que le pad se propose comme le moyen d'une écriture d'emblée collaborative ouvrant et complexifiant l'espace de travail commun.

Cette différence traduit bien une évolution des conditions et des pratiques de formation initiale des professeurs. La mastérisation de la formation (2010) a conduit à donner une place importante à une écriture de recherche dont la visée professionnalisante est plus ou moins assumée selon les trajectoires des formateurs et des formés. Ce type de production oriente désormais la formation des stagiaires alors qu'en 2009, le tuteur avait pour tâche d'inventer et de négocier des formats pour des ressources pédagogiques co-construites. La référence à des travaux théoriques ne s'imposant pas, le risque existait de laisser le travail de collaboration tourner à la simple mutualisation de ressources pour enseigner. Le bilan avait été décevant sur ce point « la grande majorité des échanges dans les forums ont servi principalement à mutualiser les pratiques, la réflexion sur ces pratiques ne se faisant qu'à la marge. » (Ahr et al., 2010, §9).

\subsection{Entretenir la réflexivité grâce à la distance}

Malgré la différence des espaces numériques utilisés, les dispositifs de formation de 2009 et 2016 ont done tenté conjointement de mettre en synergie la communication à distance et la réflexivité.

« Cette formation à distance visait à développer les interactions entre pairs et à former des praticiens réflexifs » (Ahr et al., 2010). Cette phrase tirée de l'article de 2010 conviendrait toujours ici. Naguère comme aujourd'hui, les promoteurs d'un projet de formation en ligne ont envisagé la distance comme un moyen de favoriser le retour sur des expériences d'enseignement dans une dialectique de la stabilisation et du renouvellement des pratiques.

Différents travaux théoriques justifient une telle entreprise. Collaborer à l'écrit sans la présence physique des participants suppose, selon Annie Jézégou, de maintenir une « présence à distance » nécessaire aux actions de e-learning en « communauté de recherche ${ }^{5}$ » (2010). L'effet de présence repose sur l'association de dimensions cognitive et psychoaffective. Autrement dit, collaborer réellement en ligne implique de manifester, à travers des écrits visibles de tous, un effort de rationalisation heuristique tout en maintenant une suffisante courtoisie et le souci des propositions d'autrui.

Quand il s'agit de coproduire en ligne des propositions didactiques entre professeurs débutants sous le regard de formateurs, un accueil à la fois attentif et bienveillant des écrits des autres s'avère nécessaire. Réciproquement, on peut penser que cette relation, une fois établie, favorise la réflexivité. La justification des jugements et de leurs critères, la désignation précise des gestes didactiques et le commentaire de leurs ajustements, l'examen des intentions se développeraient ainsi plus aisément.

La comparaison de deux actions de formation à distance à des moments et dans des contextes différents contribue donc à étayer l'hypothèse principale de l'étude qui sera développée ci-après. Les écritures collaboratives à distance joueraient - sous certaines conditions - un rôle favorable au processus délicat

\footnotetext{
${ }^{5}$ L'article de cette chercheuse cité en bibliographie commente et prolonge le modèle de la « community of inquiry » des professeurs canadiens Randy Garrison et Terry Anderson.
} 
de la " professionnalité émergente ». Il s'agit avec le pad d'aider les formés à " poser une analyse sur des gestes encore peu assurés, sur des compétences en cours de construction, sur des identités en transition, sur des intentions encore peu explicites » (Jorro et De Ketele, 2011, p.11).

\section{DE L'INTÉRÊT DES ÉCRITS INTERMÉDIAIRES POUR FORMER DES DÉBUTANTS RÉFLEXIFS}

\section{2 .1. Un nouveau genre d'écrit professionnel}

Depuis la mise en place des ÉSPÉ, les lauréats d'un concours d'enseignement du second degré déjà titulaires d'un master 2, sont affectés à mi-temps dans un établissement et suivent une formation allégée par rapport à ceux qui doivent achever leur master MEEF (Métiers de l'enseignement, l'éducation et de la formation). Leur formation universitaire est validée par un Travail scientifique de nature réflexive (dorénavant TSNR), cadré sur le plan national mais avec une relative latitude de mise en œuvre. Lorsqu'il s'est agi de concevoir les modalités de cet écrit, l'équipe pluricatégorielle dont sont issus les auteurs de l'article a tenu compte du fait que ces stagiaires avaient déjà réalisé un mémoire de recherche, généralement disciplinaire, et que tout l'enjeu serait de les faire entrer dans une autre forme d'écriture de recherche convoquant leur pratique et différents filtres théoriques permettant de l'analyser. Des recherches sur les mémoires professionnels en IUFM ou dans des structures francophones comparables, nous avons retenu l'idée que, pour atteindre ses objectifs de formation, le TSNR devait s'inscrire dans une temporalité compatible avec la lente évolution des questionnements chez un débutant, s'appuyer sur les premières réussites autant que difficultés rencontrées pour recentrer leur regard sur l'activité des élèves et faire alterner les modalités d'écriture individuelles et d'échange collectif (Vanhule, 2004 ; Goigoux et al., Elalouf et al. 2012).

Plus explicitement exigé que précédemment, le recueil de données relevant de l'activité professionnelle du stagiaire, leur analyse et le retour ainsi distancié sur leur propre pratique justifiaient la dénomination de travail scientifique de nature réflexive.

\section{2. Une écriture accompagnée}

S'appuyant sur les travaux ayant montré le potentiel de réflexivité des écrits de travail (Chabanne et Bucheton, 2002), l'équipe de formateurs a donc élaboré et présenté un guide prévoyant deux écrits intermédiaires alternant avec des ateliers avant la rédaction finale du TSNR. La démarche suit le modèle réflexif de Korthagen (2001, cité par Jorro, 2013) : 1. Action 2. Retour d'expérience 3. Prise de conscience des enjeux d'amélioration 4. Décision d'une nouvelle action 5. Mise en œuvre.

La première étape (novembre/décembre) s'inscrit dans le prolongement de six journées d'observation dans l'établissement du stagiaire avec un cahier des charges précis et de courts bilans intermédiaires. Il est demandé aux stagiaires de choisir deux situations d'enseignement issues de leur pratique professionnelle et visant des objectifs d'apprentissage comparables - l'une ayant donné satisfaction, l'autre n'ayant pas répondu à leurs attentes, de collecter des indices matériels sur lesquels fonder la perception des résultats obtenus et d'émettre des hypothèses sur les causes possibles de ces résultats en faisant émerger un questionnement professionnel (2 pages). La présentation orale en atelier permet d'envisager une première réflexion sur les critères mobilisés par les stagiaires pour porter un jugement sur leur pratique. Ceux-ci sont confrontés à l'analyse des documents apportés par les stagiaires (productions d'élèves, textes lus, descriptif de séquence, etc.).

Lors de la seconde étape (février mars), les stagiaires sont invités à se centrer sur l'une des activités et à approfondir leur questionnement, avant de concevoir une nouvelle démarche d'enseignement qu'ils soumettent à leur tuteur et qui fait l'objet d'une nouvelle présentation en atelier. Vient enfin la mise en œuvre de cette dernière démarche et son analyse en atelier. C'est sur la base de ces écrits intermédiaires que les stagiaires rédigent un texte d'une quinzaine de pages s'appuyant sur un corpus réuni en annexes. 


\section{COMMENT ET POURQUOI UTILISER L'ECRITURE COLLABORATIVE À DISTANCE POUR ACCOMPAGNER LA RÉDACTION DU TSNR ?}

Pour la rédaction de cet écrit, les professeurs stagiaires de lettres de l'ÉSPÉ de l'académie Versailles sont répartis en groupes d'une douzaine de personnes et accompagnés par leur tuteur universitaire qui les évalue également au cours des visites au sein de leurs classes. À titre expérimental, un suivi hybride a fait alterner des échanges en présentiel et à distance, pour accompagner la rédaction du TSNR 6 .

Les formateurs sont partis de l'idée que les stagiaires pourraient utiliser l'écriture collaborative pour prendre conscience de leurs gestes professionnels afin de les ajuster au plus près des besoins réels des élèves, mais aussi pour construire un groupe de pairs sur lequel s'appuyer pour réfléchir de manière distanciée et remettre en question leurs pratiques.

Cependant afin d'observer comment se construit leur identité professionnelle et l'ébauche d'une réflexivité à travers l'écriture et comment l'interaction avec les pairs et avec la formatrice joue son rôle dans cette construction, il fallait autant que possible rendre visibles les différents états de l'écriture et la dynamique des interactions. L'éditeur de textes collaboratifs en temps réel ETHERPAD, mis à disposition des professeurs de l'académie de Versailles, est apparu comme une solution pertinente pour la mise en place d'une écriture collaborative permettant d'accompagner la rédaction du TSNR. Mais cela nous a amené à nous interroger : en quoi l'écriture collaborative et en particulier ses modalités d'interactions écrites à distance sur ETHERPAD invitent-elles à la réécriture et in fine quel rôle joue la pluralité d'interventions questionnantes sur un même texte dans le déplacement des professeurs stagiaires vers plus de réflexivité ?

\section{1. Un dispositif hybride}

Après une exposition en présentiel des objectifs du TSNR, une prise en main du logiciel et une présentation par les stagiaires de leur projet dans un climat de confiance et d'intérêt mutuel, chacun a créé son $\mathrm{pad}^{7}$ où il décrivait et analysait sa ou ses séances et éventuellement insérait des liens permettant de voir les productions de ses élèves.

Les deux séances suivantes d'accompagnement à la rédaction individuelle de chaque TSNR ont consisté en échanges synchrones : les 6 à 7 participants de chaque sous-groupe étaient connectés tous au même moment sur le même pad, chaque production étant examinée à tour de rôle. L'utilisation d'ETHERPAD s'est donc inscrite dans l'emploi du temps des stagiaires comme une séance de formation à distance avec un calendrier précisant les séances d'échanges et de retours sur les écrits produits. Les pads des autres membres du groupe devaient être tous lus en amont de la séance. Outre cette organisation assez stricte, le tuteur proposait également un accompagnement pédagogique proactif, anticipant sur la réception des pads par les autres stagiaires pour faciliter et guider les interactions par une série de questions ouvertes et de réflexions. À l'heure programmée, dix minutes étaient consacrées à chaque pad, le tuteur se chargeant le plus souvent d'animer la séance en synthétisant les contributions, en invitant les participants à confronter leurs points de vue, mais aussi en sollicitant chacun, en valorisant le travail fourni et les interactions, en régulant le temps passé... pour maintenir des relations socio-affectives positives garantissant le climat le plus propice aux progrès de l'ensemble du groupe. Les recherches sur les modalités d'interventions tutorales dans le travail à distance (Quintin, 2011) concluent en effet sur l'importance du tutorat non seulement pour produire des interactions riches et positives, mais aussi pour atteindre les buts fixés.

\footnotetext{
${ }^{6}$ La formation en master MEEF2 Lettres est discutée au sein du département de Lettres de l'ÉSPÉ et déclinée dans chaque lieu de formation. L'expérimentation décrite ici a été mise en œuvre à l'Université Paris OuestNanterre La Défense.

${ }^{7}$ L'écran principal, le pad, est réservé aux textes produits. Lorsqu'un participant intervient, ce qu'il écrit apparait immédiatement dans la couleur qu'il a choisie (ou que le logiciel détermine pour lui), ce qui permet d'identifier les auteurs. L'espace de communication annexe, le « chat », à droite de l'écran offre la possibilité de commenter ce qui s'écrit sur la page principale.
} 


\section{2. Traces de réflexivité}

Puisque nous possédons des traces de ces échanges, il nous a semblé intéressant d'aller au-delà d'une simple analyse quantitative des interventions qui aurait de plus l'inconvénient de ne pas rendre compte de l'activité réelle et de l'investissement des participants. En effet la dynamique des échanges au sein du groupe repose sur une large palette d'attitudes allant de la lecture sans intervention à des prises de parole médiées plus ou moins pertinentes. Depover et Quintin (2011) analysent, dans les formations à distance, l'attitude de ceux qu'ils appellent des « lurkers " ", qui profitent des messages laissés par les autres sans pour autant contribuer de manière active à la recherche collective. Il s'agit de trouver les moyens de les « réveiller ». Sur ETHERPAD, tous les présents étant identifiés, le tuteur peut les interpeler nommément ; de plus, tous savent que leur participation est une des conditions requises pour valider leur parcours. Néanmoins certaines contributions s'apparentent de fort près à cette attitude. Ainsi Romain multiplie les " super ! ", " bonne idée », " je vais le faire », il se montre bon camarade en félicitant les uns et les autres, mais lui-même ne débat pas : il utilise les échanges non pas comme un moyen d'exercer sa réflexivité, mais comme un réservoir d'idées, de bonnes pratiques à imiter, sans en comprendre la logique profonde. Son TSNR témoigne de ce manque de discernement qui le conduit à appliquer des « recettes » de façon inadaptée sans tenir compte des élèves, du contexte et du savoir enseigné.

\section{3. Évaluer la réflexibilité à travers les interactions}

Si l'absence d'engagement réel est très visible, il est plus difficile de mesurer avec précision le niveau de réflexivité et les fluctuations opérées tantôt pour se replier sur des réflexions sécurisantes parce que mieux maitrisées, tantôt pour prendre le risque d'innover qui amène à penser autrement et à déplacer son angle de vue. Dans la cinquantaine de pads réalisés par les stagiaires de Lettres durant l'année scolaire 2015-2016, nous avons tenté d'évaluer des niveaux de réflexivité manifestés par l'interaction entre les stagiaires, en les articulant aux capacités attendues, selon trois dimensions :

- Ce qu'ils sont capables de dire de leur propre action (constats, explications, justifications).

- Ce qu'ils disent être capables de faire faire aux élèves

- Ce qu'ils sont capables d'évaluer comme acquis chez leurs élèves.

Tableau 1 : croisement des niveaux de réflexivité avec les capacités attendues

\begin{tabular}{|c|c|c|c|}
\hline $\begin{array}{l}\text { Discours sur } \\
\text { ce qu'ils sont } \\
\text { capables... }\end{array}$ & $\begin{array}{l}\text { de dire de leur propre } \\
\text { action }\end{array}$ & $\begin{array}{l}\text { de faire faire aux } \\
\text { élèves }\end{array}$ & $\begin{array}{l}\text { d'évaluer comme } \\
\text { acquis chez leurs } \\
\text { élèves }\end{array}$ \\
\hline Niveau 1 de réflexivité & $\begin{array}{l}\text { Rendre compte, } \\
\text { décrire et expliquer ce } \\
\text { qui a été fait en cours } \\
\text { par le professeur de } \\
\text { manière précise et } \\
\text { contextualisée. }\end{array}$ & $\begin{array}{l}\text { Faire entrer les élèves } \\
\text { dans une tâche donnée. }\end{array}$ & $\begin{array}{l}\text { Percevoir les acquis de } \\
\text { façon globale en } \\
\text { s'appuyant sur les } \\
\text { interventions du } \\
\text { groupe d'élèves le plus } \\
\text { actif de la classe. }\end{array}$ \\
\hline Niveau 2 de réflexivité & $\begin{array}{l}\text { À l'issue de } \\
\text { l'observation de sa } \\
\text { propre pratique, } \\
\text { repérer les décalages, } \\
\text { les ajustements (ou } \\
\text { leur absence) entre la }\end{array}$ & $\begin{array}{l}\text { Étayer le travail des } \\
\text { élèves pour qu'ils } \\
\text { parviennent au bout de } \\
\text { la tâche donnée. }\end{array}$ & $\begin{array}{l}\text { Discerner le niveau } \\
\text { acquis par la plupart } \\
\text { des élèves et les } \\
\text { éventuelles }\end{array}$ \\
\hline
\end{tabular}

\footnotetext{
${ }^{8}$ Lurkers : rôdeurs, observateurs passifs.
} 


\begin{tabular}{|c|c|c|c|}
\hline & $\begin{array}{l}\text { planification prévue et } \\
\text { ce qui a été réalisé. }\end{array}$ & & $\begin{array}{l}\text { résistances/difficultés } \\
\text { de quelques-uns. }\end{array}$ \\
\hline Niveau 3 de réflexivité & $\begin{array}{l}\text { Revenir sur les choix } \\
\text { didactiques qui sont à } \\
\text { l'origine du } \\
\text { déroulement de la } \\
\text { leçon pour les } \\
\text { décomposer, les } \\
\text { expliciter, en } \\
\text { comprendre l'impact. }\end{array}$ & $\begin{array}{l}\text { Proposer d'autres } \\
\text { activités possibles ou } \\
\text { un aménagement dans } \\
\text { les activités prévues } \\
\text { pour construire des } \\
\text { apprentissages plus } \\
\text { efficaces et/ou } \\
\text { permettant une } \\
\text { progression, une } \\
\text { adaptation aux } \\
\text { difficultés des élèves. }\end{array}$ & $\begin{array}{l}\text { Décomposer les } \\
\text { paliers d'apprentissage } \\
\text { par lesquels passent les } \\
\text { élèves lorsqu'ils } \\
\text { construisent les } \\
\text { compétences visées. }\end{array}$ \\
\hline Niveau 4 de réflexivité & $\begin{array}{l}\text { Réfléchir à ce qui a } \\
\text { provoqué les décalages } \\
\text { entre les choix initiaux } \\
\text { et la mise en œuvre en } \\
\text { s'appuyant sur des } \\
\text { lectures, des } \\
\text { expériences. }\end{array}$ & $\begin{array}{l}\text { Opérer des transferts, } \\
\text { des ajustements d'une } \\
\text { activité à l'autre } \\
\text { permettant de voir les } \\
\text { principes à l'œuvre } \\
\text { dans une action. } \\
\text { Proposer des exercices } \\
\text { tenant compte de } 2 \text { ou } \\
3 \text { niveaux différents au } \\
\text { sein d'une classe. }\end{array}$ & $\begin{array}{l}\text { Analyser la manière } \\
\text { dont chaque élève a } \\
\text { construit ses } \\
\text { compétences pour le } \\
\text { guider, personnaliser } \\
\text { son apprentissage. }\end{array}$ \\
\hline $\begin{array}{l}\text { Praticien réflexif } \\
\text { expérimenté }\end{array}$ & $\begin{array}{l}\text { Questionner ses choix } \\
\text { didactiques et faire le } \\
\text { lien avec les théories } \\
\text { sous-jacentes qu'ils } \\
\text { mettent en œuvre. }\end{array}$ & $\begin{array}{l}\text { Décontextualiser son } \\
\text { action de manière à la } \\
\text { voir comme un } \\
\text { principe qui comprend } \\
\text { des variantes diverses } \\
\text { à ajuster à la situation } \\
\text { telle qu'elle se } \\
\text { présente. }\end{array}$ & $\begin{array}{l}\text { Permettre à chaque } \\
\text { élève de construire son } \\
\text { propre parcours } \\
\text { d'apprentissage et son } \\
\text { auto-évaluation. }\end{array}$ \\
\hline
\end{tabular}

\section{4. Analyser les niveaux de réflexivité au sein d'un exemple}

Tableau 2 : Exemple d'échanges tiré du pad de Marie'

\begin{tabular}{|l|l|c|}
\hline \multicolumn{1}{|c|}{ Interactions } & \multicolumn{1}{|c|}{ Indicateurs } & Niveau \\
\hline $\begin{array}{l}\text { Marie : J'ai encore du mal à déterminer une } \\
\text { problématique }{ }^{\mathbf{0} \mathbf{0}} \text { vraiment précise et didactique, qui } \\
\text { se construise autour de la question des compétences et } \\
\text { des acquis. }\end{array}$ & $\begin{array}{l}\text { Énoncer une difficulté } \\
\text { didactique }\end{array}$ & $\mathbf{1}$ \\
\hline
\end{tabular}

\footnotetext{
${ }^{9}$ La graphie et la typographie des scripteurs sont respectées.

${ }^{10}$ Les caractères gras signalent la circulation des expressions d'une intervention à l'autre, pas nécessairement contigües ou au sein d'une même intervention. Les termes auvre, originalité/inventivité, texte, et littérature sont soulignés parce qu'ils n'apparaissent qu'une fois alors qu'ils relèvent du thème traité.
} 


\begin{tabular}{|c|c|c|}
\hline $\begin{array}{l}\text { Hélö̈se : Il me semble que ces trois questions/enjeux } \\
\text { que tu poses sont trois problématiques possibles - } \\
\text { comment faire écrire tous les élèves ? Comment } \\
\text { élaborer une œuvre commune tout en respectant } \\
\text { l'originalité/inventivité de chacun? Que gagne la } \\
\text { production écrite dans ces allers-retours entre travail } \\
\text { collectif et travail individuel? } \\
\text { La plus précise, la plus facile à observer et la plus } \\
\text { intéressante à mon sens étant la } 3^{\mathrm{e}} \text {. }\end{array}$ & $\begin{array}{l}\text { Reformuler le questionnement, } \\
\text { modaliser. Problématiser par } \\
\text { ajustements successifs. }\end{array}$ & 2 \\
\hline $\begin{array}{l}\text { Noémie : Tu te sers de l'écrit pour souder la classe, } \\
\text { mais aussi de la classe pour permettre une meilleure } \\
\text { acquisition d'un travail écrit. Je ne m'y connais pas en } \\
\text { didactique, mais il y a quelque chose à travailler dans } \\
\text { le domaine du groupe classe, peut-être : " comment } \\
\text { tirer profit du groupe-classe pour consolider des } \\
\text { connaissances une bonne idée! » }\end{array}$ & $\begin{array}{l}\text { Questionner l'organisation } \\
\text { pédagogique, analyser ses } \\
\text { effets, proposer des } \\
\text { modulations. }\end{array}$ & 3 \\
\hline \multicolumn{3}{|l|}{$\begin{array}{l}\text { Tutrice : Utiliser le groupe classe comme régulateur } \\
\text { paraît une excellente idée. }\end{array}$} \\
\hline $\begin{array}{l}\text { Héloïse : Aurais-tu des éléments de réponse pour cette } \\
\text { troisième "problématique"? Qu'as-tu pu observer? }\end{array}$ & $\begin{array}{l}\text { Demander des prises } \\
\text { d'information. }\end{array}$ & 2 \\
\hline $\begin{array}{l}\text { Marie : Ma principale observation est que le travail } \\
\text { gagne de façon évidente en étoffement: les secondes } \\
\text { ayant beaucoup de mal à produire un texte long. }\end{array}$ & $\begin{array}{l}\text { Formuler des observations sur } \\
\text { les productions des élèves. }\end{array}$ & 2 \\
\hline Romain : Ok & & 1 \\
\hline $\begin{array}{l}\text { Héloïse : Peut-être gagnent-ils en confiance aussi? Il } \\
\text { faudrait que tu puisses observer les élèves plutôt petits } \\
\text { parleurs et te rendre compte de leur production dans } \\
\text { un travail de groupe. Est-ce que cela les met en } \\
\text { confiance? est-ce que cela les pousse au travail, libère } \\
\text { des idées? Ou au contraire, se reposent-ils sur le groupe } \\
\text { pour en faire moins? }\end{array}$ & $\begin{array}{l}\text { Questionner l'organisation } \\
\text { pédagogique, analyser ses } \\
\text { effets, proposer des } \\
\text { modulations. }\end{array}$ & 3 \\
\hline $\begin{array}{l}\text { Marie : Oui c'est une bonne piste, j'ai constaté de façon } \\
\text { générale que cela les motivait à produire, d'autant que } \\
\text { chacun devait arriver au préalable avec une production } \\
\text { personnelle (nécessité de présenter qch au groupe=une } \\
\text { bonne motivation). }\end{array}$ & $\begin{array}{l}\text { Formuler des observations sur } \\
\text { les productions des élèves et en } \\
\text { inférer ce qui les motive. }\end{array}$ & 2 \\
\hline $\begin{array}{l}\text { Tiphaine : Y a-t-il un modérateur qui dit "stop, on } \\
\text { prend tel phrase" quand ils ne sont pas d'accord ? Ou } \\
\text { ils doivent se mettre d'accord seuls? (oups désolée j'ai } \\
\text { effacé ta phrase en effaçant la mienne...) }\end{array}$ & $\begin{array}{l}\text { Questionner l'organisation } \\
\text { pédagogique, analyser ses } \\
\text { effets, proposer des } \\
\text { modulations. }\end{array}$ & 3 \\
\hline $\begin{array}{l}\text { Marie : Peut-être à tort, je n'ai pas mis en place de } \\
\text { "rôles" définis dans chaque groupe: ils doivent donc se } \\
\text { mettre d'accord tous seuls: avec le risque que les } \\
\text { "fortes têtes" l'emportent... }\end{array}$ & $\begin{array}{l}\text { Mettre en relation } \\
\text { l'apprentissage visé et les } \\
\text { modalités choisies. }\end{array}$ & 2 \\
\hline $\begin{array}{l}\text { Tiphaine : Ce n'est pas nécessairement à tort, après } \\
\text { tout ils sont au lycée, il n'y a peut-être pas besoin de } \\
\text { modérateur... Disons que tu travailles l'âme des } \\
\text { "leaders" ;P }\end{array}$ & $\begin{array}{l}\text { Mettre en relation } \\
\text { l'apprentissage visé et les } \\
\text { modalités choisies. }\end{array}$ & 2 \\
\hline \multicolumn{3}{|l|}{ Romain : mouais...;-)= } \\
\hline Marie : j'ai essayé d'équilibrer les groupes... & Expliquer ce qui a été fait. & 1 \\
\hline
\end{tabular}




\begin{tabular}{|c|c|c|}
\hline $\begin{array}{l}\text { Tutrice : Il faut absolument que je vous envoie ce que } \\
\text { j'ai préparé sur le travail de groupe par Astolfi, je ferai } \\
\text { un envoi pour tous. }\end{array}$ & & \\
\hline $\begin{array}{l}\text { Romain : ok merci! } \\
2 \text { choses hors sujet: il serait bon d'avoir encore si c'était } \\
\text { possible des cours de M. P. M. là dessus. Ensuite, les } \\
\text { pastiches, mais ça n a rien à voir avec les dissert! leur } \\
\text { donnent du goût pr l'écriture. sorry pr mes hors sujet. } \\
\text { La littérature contemporaine au lycée est à faire } \\
\text { connaitre davantage! Kerangal par ex, et la façon } \\
\text { nouvelle de mettre en dialogue! }\end{array}$ & $\begin{array}{l}\text { Proposer d'autres supports : des } \\
\text { références littéraires ou } \\
\text { artistiques liées au thème, au } \\
\text { parcours, au niveau d'étude... }\end{array}$ & 1 \\
\hline $\begin{array}{l}\text { Héloïse : Ok du coup ça te fait déjà deux éléments de } \\
\text { réponse/ parties pour ton mémoire. } \\
\text { Que gagne la production écrite dans ces allers- } \\
\text { retours entre travail collectif et travail individuel? } \\
1 \text { - Elle gagne en densité de production. } \\
2 \text { - Elle gagne en quantité d'élèves mis au travail, } \\
\text { puisqu'elle contribue à libérer la parole des plus } \\
\text { timides. }\end{array}$ & $\begin{array}{l}\text { Cibler avec précision le } \\
\text { problème didactique posé et } \\
\text { apporter des éléments de } \\
\text { réponse. }\end{array}$ & 4 \\
\hline $\begin{array}{l}\text { Marie : Oui ce sont deux pistes très intéressantes! } \\
\text { merci! et de façon un peu "basique" cela permet aussi } \\
\text { de rectifier la correction de l'expression... }\end{array}$ & $\begin{array}{l}\text { Identifier un autre effet potentiel } \\
\text { des choix didactiques. }\end{array}$ & 3 \\
\hline $\begin{array}{l}\text { Tutrice : C'est ce que dit Bucheton dans ses travaux, les } \\
\text { écrits ne sont pas forcément "meilleurs" ou plus longs } \\
\text { ou plus corrects du point de vue de la langue, ils sont } \\
\text { surtout plus "épais". }\end{array}$ & & \\
\hline Marie : Oui ils sont plus denses en fait. & $\begin{array}{l}\text { Reformuler une caractéristique } \\
\text { des productions d'élèves liée au } \\
\text { dispositif. }\end{array}$ & 3 \\
\hline $\begin{array}{l}\text { Tutrice : Je crois que la correction de l'expression est } \\
\text { à garder pour un autre moment que l'on pourrait appeler } \\
\text { "travail de révision" ou de "nettoyage", c'est comme ça } \\
\text { que j'en parle aux élèves. }\end{array}$ & & \\
\hline $\begin{array}{l}\text { Marie : Plus exactement pas l'expression, la } \\
\text { correction de la langue, toutes les erreurs d'accord, de } \\
\text { conjugaison.... } \\
\text { Oui afin de ne pas mobiliser trop de compétences à la } \\
\text { fois? }\end{array}$ & $\begin{array}{l}\text { Reformuler en prenant en } \\
\text { compte une objection et en } \\
\text { cherchant une justification. }\end{array}$ & 3 \\
\hline
\end{tabular}

Le débat porte sur une dissertation que Marie veut faire écrire en groupe à partir des premiers essais individuels des élèves. Grâce aux interventions d'Héloïse et de Tiphaine qui vont reformuler sa problématique, l'inviter à creuser le sens de ses activités, à développer ce qu'elle a perçu intuitivement, et finalement proposer une hiérarchisation de ses constats, on perçoit la progression de la réflexion qui se diversifie et se hisse progressivement à un niveau de réflexivité plus élevé. La qualité de l'échange collectif est telle qu'on a l'impression que tous avancent et approfondissent leur pensée malgré quelques interactions qui dévient. La permanence de ces écrits intermédiaires à l'écran permet de constater de quelle manière les propositions, questions, commentaires, confrontations, contradictions, détours, médiations... des intervenants postés sur le pad aident à fixer, relancer, épaissir la pensée, mais aussi comment le fait même de lire et commenter, écrire sur les écrits des autres fait rebondir les idées, aide à les définir, les ajuster, les clarifier. Les reprises d'unités lexicales d'une intervention à l'autre (en gras) le manifestent.

\section{Pour conclure}


Ainsi accompagnée, la pratique réflexive nous parait pouvoir être démultipliée et comprise autrement que comme un idéal hors de portée des débutants. Les pairs en réagissant tendent autant de miroirs à celui qui écrit et ouvrent un espace de pensée et de « brouillonnements » (Bucheton, 2014) qui aide à se voir autrement et à se déplacer. De plus, en étant tantôt lecteurs, tantôt scripteurs, les participants acquièrent la souplesse intellectuelle permettant l'analyse distanciée de leurs propres travaux, ils ébauchent et affinent une posture réflexive qui va les aider à rédiger la version finale de leur TSNR.

Du côté de la tutrice, le «Je ne m'y connais pas en didactique » (Noémie) et la référence aux cours donnés à l'ÉSPÉ ou à des lectures bien intégrées (par exemple la référence aux petits parleurs chez Héloïse) sont une invitation à installer de nouveaux savoirs : les participants sont mûrs pour accepter de se plonger dans des lectures didactiques qui vont les confronter à une démarche de chercheur, d'où l'annonce d'envois par courriel de références d'articles et d'extraits qui suivront l'échange. Ainsi ce travail d'écriture à distance sur ETHERPAD a tenu ses promesses au sens où il a permis à nos stagiaires d'exercer leur réflexivité sous le regard d'autrui dans un environnement sécurisant. Gageons qu'il les a aussi ouverts à une réflexion sur le potentiel pédagogique de l'ENT, rejoignant ainsi les objectifs du dispositif de 2009.

Marie-Laure Elalouf, Stéphanie Martel, Pierre Moinard

ÉSPÉ de l'académie de Versailles,

Université de Cergy-Pontoise, F-95100

ÉMA (École, Mutations, Apprentissage), ÉA 4507

\section{Références bibliographiques}

AHR, S., CZARNY, N., FEKI, H., MOINARD, P. 2010. « L'espace numérique collaboratif : un lieu pour former et se former, pour enseigner et apprendre », dans $E L A, 4 / 2010$ (n $\left.{ }^{\circ} 160\right)$, p. 465-477 URL : www.cairn.info/revue-ela-2010-4-page-465.htm.

BUCHETON D., ALEXANDRE D., JURADO M., 2014. Refonder l'enseignement de l'écriture, Retz

CHABANNE, J.-C. \& BUCHETON, D. 2002 : Parler et écrire pour penser, apprendre et se construire. L'écrit et l'oral réflexifs, Paris, PUF.

DE KETELE, J.-M., JORRO, A. 2011. (dir.) La professionnalité émergente : quelle reconnaissance? Bruxelles, De Boeck, $192 \mathrm{p}$.

DEPOVER C., DE LIEVRE B., PERAYA D., QUINTIN J.-J., JAILLET A. 2011. Le tutorat en formation à distance, Bruxelles, De Boeck, $288 \mathrm{p}$.

ELALOUF, M.-L., BEAUMANOIR-SECQ, M., BORNAZ, S., FORT, P.-L. (2012) : « Enjeux de la constitution de corpus dans les écrits professionnels et de recherche du master " éducation et formation » : le cas de la didactique du français », Les didactiques en question(s). Bruxelles, De Boeck, pp. 382-403.

GOIGOUX, R., RIA, L. \& TOCZEK-CAPELLE, M.C. 2009. Les parcours de formation des enseignants débutants, Presses de l'Université Blaise Pascal.

JORRO, A. 2013. «L'accompagnement des enseignants dans l'activité évaluative face à des situations de production écrite », dans RFLA, Vol. XVIII-1, pp. 107-116.

KORTHAGEN, F. A. J. 2001. « Linking practice and theory : the pedagogy of realistic teacher education ». Paper presented at the Annual Meeting of the American Educational Research, Association, Seattle.

QUINTIN, J.-J. 2011. «L'efficacité des modalités d'intervention tutorale et leurs effets sur le climat socio-relationnel des groupes restreints » in DEPOVER C., DE LIEVRE B., PERAYA D., QUINTIN J.-J., JAILLET A., Le tutorat en formation à distance. Bruxelles, De Boeck, pp. 61-86.

JEZEGOU, A. 2010. « Community of Inquiry en e-learning : à propos du modèle de Garrison et d'Anderson ", dans JOURNAL OF DISTANCE EDUCATION/REVUE DE L'ÉDUCATION À DISTANCE, 24 (2), pp.1-18. 
VANHULE, S. 2004. "L'écriture réflexive, une inlassable transformation sociale de soi », Repères $\mathrm{n}^{\circ} 30$, Les pratiques langagières en formation initiale et continue. Paris, INRP, pp. 13-31. 